\title{
Avaliação da atividade leishmanicida in vitro de plantas medicinais
}

\author{
Jeamile L. Bezerra1, Graciomar C. Costa², Thaiana C. Lopes ${ }^{2}$, Izabelle C.D.S. Carvalho1', \\ Fernando J. Patrício², Sanara M. Sousa ${ }^{2}$, Flávia M.M. Amaral ${ }^{2}$, José Manuel M. Rebelo \\ Rosane N.M. Guerra ${ }^{2}$, Maria Nilce S. Ribeiro ${ }^{1}$, Flávia R.F. Nascimento ${ }^{2 *}$ \\ ${ }^{1}$ Laboratório de Farmacognosia, Departamento de Farmácia, Universidade Federal do Maranhão, \\ Campus do Bacanga, Av. dos Portugueses s/n, 65085-580, São Luís, Maranhão, Brasil, \\ ${ }^{2}$ Laboratório de Imunofisiologia, Departamento de Patologia, Universidade Federal do Maranhão, \\ Campus do Bacanga, Av. dos Portugueses s/n, 65085-580, São Luís, Maranhão, Brasil, \\ ${ }^{3}$ Laboratório de Entomologia e Vetores, Departamento de Biologia, Praça da Madre Deus, 2 , \\ 65025-580, São Luís, Maranhão, Brasil
}

\begin{abstract}
RESUMO: Na busca de novos agentes leishmanicidas, avaliamos em culturas de promastigotas de Leishmania amazonensis o efeito dos extratos hidroalcoólicos das folhas de Tephrosia cinerea (L.) Pers. (Fabaceae), Dichorisandra sp (Commelinaceae), Syzygium jambolanum DC. (Myrtaceae), Julocroton triqueter (Lam.) Didr. var. triqueter (Euphorbiaceae), Passiflora edulis Sims (Passifloraceae), Cecropia sp (Cecropiaceae), Chenopodium ambrosioides L. (Chenopodiaceae), Pedilanhus tithymaloides (L.) Poit (Euphorbiaceae), Peristrophe angustifolia Nees (Acanthaceae) e o extrato aquoso do mesocarpo de Orbignya phalerata Mart. (Arecaceae). As promastigotas de Leishmania amazonensis foram cultivadas em presença de 31,$3 ; 63,5 ; 125,0 ; 250,0$ e $500,0 \mu \mathrm{g} / \mathrm{mL}$ dos extratos por 24 horas. Ao final desse período foi calculada a concentração inibitória do crescimento $\left(\mathrm{CI}_{50}\right)$ em relação às culturas não tratadas com os extratos. Os extratos das espécies $J$. triqueter, Dichorisandra sp e $T$. cinerea apresentaram maior eficácia em induzir a morte das promastigotas, com $\mathrm{CI}_{50}$ de 29,5;32,9 e 43,6 $\mu \mathrm{g} / \mathrm{mL}$, respectivamente. P. edulis, C. ambrosioides e $S$. jambolanum apresentaram eficácia moderada com $\mathrm{CI}_{50}$ de 150,$1 ; 151,9$ e $166,6 \mu \mathrm{g} / \mathrm{mL}$, respectivamente. P. tithymaloides e O. phalerata apresentaram baixo efeito leishmanicida com $\mathrm{CI}_{50}>500 \mu \mathrm{g} / \mathrm{mL}$, enquanto Peristrophe angustifolia e Cecropia sp não apresentaram efeito. Dessa forma, dos dez extratos testados, três apresentaram uma expressiva atividade leishmanicida in vitro.
\end{abstract}

Unitermos: Leishmania amazonensis, atividade leishmanicida, plantas medicinais.

\begin{abstract}
Evaluation of the in vitro leishmanicidal activity of medicinal plants". Searching for new leishmanicidal agents, promastigotes forms of L. amazonensis were cultured with the hydroalcoholic extracts obtained from the Tephrosia cinerea (L.) Pers. (Fabaceae), Dichorisandra sp (Commelinaceae), Syzygium jambolanum DC. (Myrtaceae), Julocroton triqueter (Lam.) Didr. var. triqueter (Euphorbiaceae), Passiflora edulis Sims (Passifloraceae), Cecropia sp (Cecropiaceae), Chenopodium ambrosioides L. (Chenopodiaceae), Pedilanhus tithymaloides (L.) Poit (Euphorbiaceae), Peristrophe angustifolia Nees (Acanthaceae) leaves and the aqueous extract obtained from the Orbignya phalerata Mart. (Arecaceae) mesocarp flour. The in vitro assay was performed with promastigotes incubated during 24 hours with 31.3, 62.5, 125.0, 250.0 and 500.0 $\mu \mathrm{g} / \mathrm{mL}$ of each extract. Then the inhibitory concentration of the parasite growth $\left(\mathrm{IC}_{50}\right)$ was determined. The effectiveness of J. triqueter, Dichorisandra sp and T. cinerea hydroalcoholic extracts to induce promastigotes death was intense since the $\mathrm{IC}_{50}$ were $29.5 ; 32.9$ and $43.6 \mu \mathrm{g} / \mathrm{mL}$, respectively. P. edulis, C. ambrosioides and $S$. jambolanum extracts had moderated effectiveness since the $\mathrm{IC}_{50}$ were $150.1 ; 151.9$ and $166.6 \mu \mathrm{g} / \mathrm{mL}$, respectively. $P$. tithymaloides and $O$. phalerata extracts showed a low efficacy in comparison with $\mathrm{IC}_{50}>500$ $\mu \mathrm{g} / \mathrm{mL}$. Peristrophe angustifolia and Cecropia sp extracts had no leishmanicidal effect. Thus, three of the ten extracts that were tested showed a significant in vitro leishmanicidal activity.
\end{abstract}

Keywords: Leishmania amazonensis, leishmanicidal activity, medicinal plants.

\section{INTRODUÇÃO}

A leishmaniose é uma doença infecciosa, não contagiosa, causada por protozoários do gênero Leishmania, endêmica ao redor do mundo, que afeta mais de 12 milhões de pessoas mundialmente e apresenta alta endemicidade, morbidade e mortalidade em populações da África, Ásia e América Latina (WHO, 2002, Dujardin, 2006).
No Brasil ocorre em todo o território, tanto a Leishmaniose visceral (LV) como a Leishmaniose Tegumentar Americana (LTA), as quais são endêmicas nas regiões Norte e Nordeste, devido principalmente às características econômicas e culturais dessas populações, predominando principalmente nos estados da Bahia, Ceará, Piauí e Maranhão (Ministério da Saúde, 2000; Rath et al., 2003).

O Estado Maranhão apresenta grande incidência 
de leishmanioses em $88 \%$ de seus municípios, sendo o município de Buriticupu, situado na região pré-amazônica do Estado, o que apresenta os mais altos coeficientes de detecção da LTA (Silva et al., 1979; Costa et al., 1998; Rebêlo et al., 2001a,b; Rebêlo et al., 2000 a,b; Martins, 2002; Caldas et al., 2002).

$\mathrm{O}$ tratamento das leishmanioses é feito à base de antimoniais pentavalentes, anfotericina B e pentamidinas, as quais são tóxicas, de custo elevado, difícil administração e podem causar resistência ao parasito (Rath et al., 2003; Croft; Coombs 2003). Novas drogas como miltefosine, um hexadecil-fosfocolina, têm demonstrado ser efetivo para o tratamento de LV, muito embora apresentem também alta toxicidade (Sindermann et al., 2004). A internação prolongada e os efeitos adversos como alterações cardíacas, renais, pancreáticas e hepáticas dificultam a adesão ao tratamento. Considerando as dificuldades de tratamento e a ausência de vacinas, há urgência na busca de novas drogas terapêuticas dentre as quais se incluem os fitoterápicos (Carvalho; Ferreira, 2001; Paula et al., 2003; Nakamura et al., 2006).

De acordo com a Organização Mundial de Saúde (OMS), as espécies vegetais são a melhor e maior fonte de fármacos para humanidade. Estudos etnobotânicos têm demonstrado o uso popular de plantas no tratamento das leishmanioses tanto por via oral, como na aplicação tópica sobre as lesões cutâneas (França et al., 1993; 1996; Mathias; Emily, 1993; Silva et al., 1995; Moreira et al., 2002). Muitos vegetais apresentam em sua composição substâncias das classes dos alcalóides, terpenos, lignanas, chalconas, flavonóides e lactonas sesquiterpênicas, compostos descritos na literatura como eficazes na atividade leishmanicida e/ou anti-Leishmania (Iwu et al., 1994; Queiroz et al., 1996; Torres-Santos, 1999; Kam et al., 1999; Rocha et al., 2005).

Este trabalho avaliou o efeito leishmanicida in vitro dos extratos hidroalcoólicos de folhas das espécies Tephrosia cinerea (L.) Pers., Dichorisandra sp, Syzygium jambolanum D.C., Julocroton triqueter (Lam.) Didr. var. triqueter, Passiflora edulis Sims, Chenopodium ambrosioides L., Pedilanthus tithymaloides (L.) Poit., Peristrophe angustifolia Nees, Cecropia sp e do extrato aquoso do mesocarpo de Orbignya phalerata Mart., em formas promastigotas de Leishmania amazonensis.

\section{MATERIAL E MÉTODOS}

\section{Material vegetal}

Folhas de Tephrosia cinerea (L.) Pers. (Fabaceae), Dichorisandra sp (Commelinaceae), Syzygium jambolanum DC. (Myrtaceae), Julocroton triqueter (Lam.) Didr. var. triqueter (Euphorbiaceae), Passiflora edulis Sims (Passifloraceae), Chenopodium ambrosioides L. (Chenopodiaceae), Pedilanthus tithymaloides (L.) Poit. (Euphorbiaceae), Peristrophe angustifolia Nees (Acanthaceae) e Cecropia sp
(Cecropiaceae) foram coletadas no Campus da Universidade Federal do Maranhão (Bacanga/São Luís, MA/Brasil) no período de julho a setembro de 2004.

O pó do mesocarpo de Orbignya phalerata Mart. (Arecaceae) foi obtido comercialmente da empresa HENSA FARMA, São Luís, MA, Brasil. Este produto comercial foi previamente submetido a análise de autenticidade, integridade e pureza por testes físicoquímicos. O pó comercial apresentou similaridade em todos os aspectos botânicos e fitoquímicos testados quando comparado com o pó de mesocarpo obtido no próprio laboratório (Batista; Ribeiro, 2003). Para identificação botânica os frutos de $O$. phalerata foram coletados em Pedreiras-MA, Brasil.

As identificações botânicas foram realizadas pela Dra. Terezinha de Jesus Almeida Rêgo, no Herbário Ático Seabra, da Universidade Federal do Maranhão (UFMA), onde as exsicatas estão depositadas conforme numeração descrita na Tabela 1.

\section{Preparação dos extratos}

O pó do mesocarpo de O. phalerata $(20 \mathrm{~g})$, foi macerado em água, filtrado, obtendo-se o extrato aquoso. Os demais extratos foram preparados a partir das folhas. As folhas foram secas em temperatura ambiente e trituradas em moinho. O pó das folhas das espécies Dichorisandra sp (39,2g), T. cinerea (480g), S. jambolanum (200g), J. triqueter $(398,6 \mathrm{~g})$, P. edulis (100g), C. ambrosioides (200g), Cecropia sp (200g), P. tithymaloides $(74,8 \mathrm{~g})$, P angustifolia $(127 \mathrm{~g})$ foram então extraídas, exaustivamente, por maceração com etanol a $70 \%$. As soluções extrativas foram submetidas à filtração e, em seguida, concentradas em rotoevaporador à pressão reduzida. Os resíduos secos foram diluídos em solução tamponada de fosfato (PBS pH 7,2) a uma concentração final de $1 \mathrm{mg} / \mathrm{mL}$.

\section{Atividade leishmanicida in vitro}

As formas promastigotas de Leishmania amazonensis (MHOM/Br/90/BA125) foram cedidas pela Dra Aldina Barral do Centro de Pesquisas Gonçalo Moniz (CPQGM), da Fundação Osvaldo Cruz (FIOCRUZ), Salvador-BA.

As culturas de promastigotas foram mantidas a $26{ }^{\circ} \mathrm{C}$ em meio NNN enriquecido com RPMI 1640 , suplementado com soro fetal bovino inativado a $10 \%$ (SIGMA), L-glutamina 2mM (GIBCO) e os antibióticos penicilina $(100 \mathrm{U} / \mathrm{mL})$ e estreptomicina $(100 \mu \mathrm{g} / \mathrm{mL})$ (SIGMA). Antes de cada experimento observou-se ao microscópio a motilidade flagelar dos parasitos.

Para os ensaios, o extrato previamente obtido foi diluído cinco vezes em meio Schneider completo, obtendo-se a concentração de $1 \mathrm{mg} / \mathrm{mL}$. Deste, foram retirados $100 \mu \mathrm{L}$ os quais foram diluídos seriadamente, em placas de 96 poços de fundo chato (Costar), na 
Tabela 1. Espécies vegetais utilizadas nesse trabalho.

\begin{tabular}{llc}
\hline \multicolumn{1}{c}{ Nome científico } & Família & Exsicata \\
\hline Cecropia sp & Cecropiaceae & 1107 \\
Chenopodium ambrosioides L. & Chenopodiaceae & 1148 \\
Dichorisandra sp & Commelinaceae & 1259 \\
Julocroton triqueter (Lam.) Didr.var. triqueter & Euphorbiaceae & 1265 \\
Orbignya phalerata Mart. & Arecaceae & 1135 \\
Passiflora edulis Sims & Passifloraceae & 1155 \\
Pedilanthus tithymaloides (L.) Poit. & Euphorbiaceae & 1250 \\
Peristrophe angustifolia Nees & Acanthaceae & 1342 \\
Syzygium jambolanum DC. & Myrtaceae & 1079 \\
Tephrosia cinerea (L.) Pers & Fabaceae & 1256 \\
\hline
\end{tabular}

proporção de 1:2 em meio RPMI completo, resultando nas concentrações finais de 500, 250, 125, 62,5 e 31,25 $\mu \mathrm{g} / \mathrm{mL}$.

Em cada poço foram, então, adicionados 10 $\mu \mathrm{L}$ da suspensão contendo $5 \times 10^{6}$ formas promastigotas de L. amazonensis por $\mathrm{mL}$. Glucantime ${ }^{\circledR}$, droga de referência no tratamento das leishmanioses, foi utilizado nas mesmas concentrações dos extratos. Como controle foram utilizadas as formas promastigotas cultivadas em meio RPMI 1640 suplementado.

A atividade leishmanicida dos extratos foi avaliada pela inibição do crescimento de formas promastigotas após $24 \mathrm{~h}$ de incubação a $26{ }^{\circ} \mathrm{C}$, pela contagem do número total de promastigotas vivas, levando-se em consideração a motilidade flagelar, utilizando-se câmara de Neubauer e microscópio ótico de luz comum. A contagem foi comparada com o controle do crescimento das formas promastigotas sem os extratos. Cada concentração dos extratos foi analisada em triplicata. Os resultados foram expressos como concentração inibitória do crescimento parasitário $\left(\mathrm{CI}_{50}\right)$, baseado em Schmeda-Hirschman et al., (1996) e Camacho et al., (2003).

Tabela 2. Atividade leishmanicida dos extratos vegetais

\section{Análise estatística}

Todos os ensaios foram realizados em triplicata e repetidos pelo menos uma vez. Os resultados foram expressos como concentração inibitória do crescimento parasitário $\left(\mathrm{CI}_{50}\right)$, calculados por regressão linear.

\section{RESULTADOS E DISCUSSÃO}

Diversos compostos químicos, isolados de extratos vegetais, têm comprovada atividade leishmanicida sobre formas promastigotas e/ou amastigotas de Leishmania em ensaios in vitro. Já foi relatada a atividade leishmanicida de terpenóides (Sauvain et al., 1996; Camacho et al., 2000), aminoglicosteróides e aminosteróides (Kam et al., 1997), naftoquinonas (Fournet et al., 1992; Kayser et al., 2000), chalconas (Chen et al., 1993; Boeck et al., 2006), glicosídios iridóides (Mittal et al., 1998), flavonóides (Araújo et al., 1998), lignanas (Barata et al., 2000; Royo et al., 2003) e de alcalóides (Mahiou et al., 1994; Fournet et al, 1996; Queiroz, et al., 1996). Apesar dos vários estudos nesta área, ainda existem muitas espécies vegetais com potencial atividade leishmanicida a serem avaliadas.

\begin{tabular}{|c|c|c|}
\hline Nome Científico & $\begin{array}{l}\text { Farmacógeno/ } \\
\text { Extrato }\end{array}$ & $\begin{array}{l}\text { Atividade Leishmanicida } \\
\mathrm{CI}_{50}(\mu \mathrm{g} / \mathrm{mL})\end{array}$ \\
\hline Cecropia sp & Folhas / EH & s/ atividade \\
\hline Chenopodium ambrosioides L. & Folhas / EH & 151,9 \\
\hline Dichorisandra $\mathrm{sp}$ & Folhas / EH & 32,9 \\
\hline Julocroton triqueter (Lam.) Didr.var. triqueter & Folhas / $\mathrm{EH}^{\mathrm{a}}$ & 29,5 \\
\hline Orbignya phalerata Mart. & Mesocarpo / fruto / $\mathrm{EA}^{\mathrm{b}}$ & $>500$ \\
\hline Passiflora edulis Sims & Folhas / EH & 150,1 \\
\hline Pedilanthus tithymaloides (L.) Poit. & Folhas / EH & $>500$ \\
\hline Peristrophe angustifolia Nees & Folhas / EH & s/atividade \\
\hline Syzygium jambolanum DC. & Folhas / EH & 166,6 \\
\hline Tephrosia cinerea (L.) Pers & Folhas / EH & 43,6 \\
\hline Glucantime $\AA$. & Droga referência & 440,3 \\
\hline \multicolumn{3}{|l|}{$\begin{array}{l}{ }^{a} \text { EH - Extrato Hidroalcoólico } \\
{ }^{b} E A \text { - Extrato Aquoso }\end{array}$} \\
\hline & & $\begin{array}{l}\text { Rev. Bras. Farmacogn. } \\
\text { Braz J. Pharmacogn. } \\
\text { 16(Supl.):dez. } 2006\end{array}$ \\
\hline
\end{tabular}


Neste trabalho foi avaliado o efeito leishmanicida de dez extratos de plantas de diferentes famílias. A escolha das espécies nesse estudo foi baseada na quimiossistemática dos gêneros, no uso popular no tratamento de úlceras e na disponibilidade local.

A avaliação dos extratos das espécies mostrou que seis deles apresentaram atividade leishmanicida significativa, sendo que três espécies, $J$. triqueter, Dichorisandra sp e $T$. cinerea apresentaram maior eficácia, com base na $\mathrm{CI}_{50}(29,5 ; 32,9$ e $43,6 \mu \mathrm{g} / \mathrm{mL}$, respectivamente) como mostra a Tabela 2 . Os extratos de T. cinerea e J. triqueter var. triqueter apresentaram $100 \%$ de atividade leishmanicida nas concentrações de 500 e $250 \mu \mathrm{g} / \mathrm{mL}$, e Dichorisandra sp. apresentou $100 \%$ de atividade na maior concentração testada.

Julocroton triqueter var. triqueter, a espécie mais efetiva, é conhecida popularmente como velame, sendo encontrada desde a região Norte até a região Sudeste do Brasil. O pó das suas folhas é usado no tratamento de úlceras, sífilis e como depurativo (Pio Corrêa, 1984), porém, há poucos estudos farmacológicos e/ou químicos sobre essa espécie, muito embora já tenham sido identificados alcalóides no gênero Julocroton (Suarez et al., 2004).

Dichorisandra sp, a segunda espécie mais eficaz, é popularmente conhecida como penicilina sendo indicada popularmente para tratar infecções. Martinez et al. (1993) relataram a presença de glicosídeos de apigenina, luteolina, quercetina, 6-hidroxiluteolina e tricina em três espécies do gênero Dichorisandra. Mittra et al. (2000) demonstraram efeito inibitório in vitro dos flavonóides luteolina e quercetina sobre o crescimento de formas promastigotas e amastigotas de Leishmania donovani, uma das espécies responsáveis pela leishmaniose visceral. Esses autores também demonstraram que o tratamento in vivo, por via oral, com essas substâncias, causou uma significativa redução da carga parasitária em baços de hamsteres infectados com L. donovani.

As folhas de Tephrosia cinerea (L.) Pers., conhecida como anil bravo, são utilizadas pela população para tratar infecções, inflamações, úlceras, afecções nervosas e diarréias (Pio Corrêa, 1994). Sharma et al. (2003) demonstraram a ação anti-leishmaniat do extrato metanólico das folhas de $T$. purpurea, uma espécie medicinal indiana, frente à espécie Leishmania donovani.

Os extratos de Passiflora edulis, Chenopodium ambrosioides e Syzygium jambolanum apresentaram atividade leishmanicida menor que os outros três extratos citados anteriormente, com $\mathrm{CI}_{50}$ de 150,$1 ; 151,9$ e 166,6 $\mu \mathrm{g} / \mathrm{mL}$, respectivamente (Tabela 2). O extrato de $P$. edulis apesar de ocasionar $100 \%$ de inibição no crescimento das formas promastigotas de Leishmania amazonensis na maior dose testada, apresentou $\mathrm{CI}_{50}$ similar à detectada nos extratos de $C$. ambrosioides e $S$. jambolanum, que apresentaram respectivamente $63,6 \%$ e $88 \%$ de inibição da Leishmania amazonensis na maior dose testada.
Chenopodium ambrosioides conhecido vulgarmente como mastruz, tem sido usado no nordeste brasileiro no tratamento de helmintíases, bronquite, tuberculose, contusões, fraturas e feridas (França et al., 1996; Rêgo, 1995; Matos, 2000; Pereira et al., 2004; Morais et al., 2005; Tôrres et al., 2005). A ação antihelmíntica da planta foi associada ao alto teor de ascaridol, presente no óleo essencial das folhas (Sousa et al., 1991). Moreira et al. (1998), ao estudarem o conhecimento e usos de terapias alternativas para Leishmaniose Tegumentar Americana, em populações no município de Buriticupu, Maranhão, constataram ser comum o uso de plantas sobre as feridas leishmanióticas, dentre as quais se incluem as folhas de mastruz. O dado obtido aqui pode justificar o uso popular deste vegetal no tratamento das úlceras leishmanióticas.

Passiflora edulis conhecida como maracujá, é tradicionalmente utilizada como calmante, pela população de todos os paises ocidentais. É uma espécie cultivada no nordeste brasileiro para aproveitamento industrial do fruto (Matos, 2000). O efeito leishmanicida apresentado por esta espécie pode estar relacionado com a presença dos compostos flavonoídicos e/ou terpenóides evidenciados nas folhas desses vegetais (Dhawan et al, 2004).

Syzygium jambolanum, conhecido vulgarmente como jambolão, apresenta intensa atividade antibacteriana (Loguercio et al., 2005; Michelin et al., 2005). O extrato das folhas apresentou efeito leishmanicida moderado, o que poderia ser justificado pela presença de saponinas e taninos condensados e hidrolisáveis nas folhas (Alberton et al., 2001).

$\mathrm{O}$ extrato de Pedilanthus tithymaloides, cuja $\mathrm{CI}_{50}$ foi $>500 \mu \mathrm{g} / \mathrm{mL}$, não apresentou atividade leishmanicida tão intensa quanto o extrato de J. triqueter, apesar da espécie pertencer à família Euphorbiaceae. Da mesma forma, o extrato de $O$. phalerata apresentou atividade leishmanicida muito baixa $\left(\mathrm{CI}_{50}>500\right)$, mantendo-se na faixa de $20 \%$ de morte das promastigotas em todas as concentrações testadas.

Os extratos de Peristrophe angustifolia e de Cecropia sp não inibiram o crescimento das formas promastigotas, em nenhuma das concentrações testadas, havendo ainda um aumento do número de promastigotas viáveis, no caso do extrato de Cecropia sp, caracterizando assim um possível efeito pró-Leishmania desse extrato.

De acordo com o nosso conhecimento, este é o primeiro relato na literatura no qual os extratos das espécies aqui avaliadas são submetidos a ensaios de atividade leishmanicida. As plantas testadas são comuns no Brasil, algumas nativas, outras introduzidas e já bem aclimatadas, outras cultivadas, portanto, fáceis de serem encontradas, o que nos estimula a continuidade de estudos químicos biomonitorados na busca dos princípios ativos associados à atividade leishmanicida detectada. Esses dados sugerem que tratamentos alternativos podem ser eficientes no controle das leishmanioses. 


\section{CONCLUSÃO}

Em nossa investigação para atividade leishmanicida com dez plantas encontradas na flora maranhense, três apresentaram uma expressiva atividade in vitro para L. amazonensis. Esses resultados indicam a necessidade de estudos futuros para a avaliação do caráter citotóxico dos extratos, da atividade em infecções, in vivo, bem como o fracionamento na busca das substâncias responsáveis pela ação leishmanicida encontrada.

\section{AGRADECIMENTOS}

Ao CNPq pelo auxílio financeiro (Proc $\mathrm{n}^{\circ} \mathrm{CNPq}$ $n^{\circ} 550433 / 2001-5$ e Proc. $n^{\circ}$ 620081/2004-0 ACT), pelas bolsas PIBIC para G.C.Costa, I.C.D.S.Carvalho, F.J.Patrício, S.M. Sousa e pela bolsa de Pós-doc de R.N.M.Guerra. À Capes pela bolsa de doutorado de F.M.M Amaral. À FAPEMA pela bolsa de mestrado de J.L. Bezerra. À Dra. Aldina Barral do Centro de Pesquisas Gonçalo Moniz-FIOCRUZ/BA, por fornecer as formas promastigotas de L. amazonensis utilizadas neste estudo. À Profa. Bárbara T. Silva do Departamento de Ciências Fisiológicas/UFMA pela concessão do extrato da espécie Passiflora edulis.

\section{REFERÊNCIAS}

Alberton JR, Ribeiro A, Sacramento LVS, Franco SL, Lima MAP 2001. Caracterização farmacognóstica do jambolão (Syzygium cumini (L.) Skeels. Rev Bras Farmacogn 11: 37-50.

Araújo CAC, Alegrio LV, Leon LL 1998. Antileishmanial activity of compounds extracted and characterized from Centrolobium sclerophyllum. Phytochemistry 49:751-754.

Barata LES, Santos LS, Ferri PH, Phillipson JD, Paine A, Croft SL 2000. Anti-leishmanicidal activity of neolignans from Virola species and synthetic analogues. Phytochemistry 55: 589-595.

Batista SM, Ribeiro MNS. 2003. Controle de qualidade de Orbignya phalerata Mart. In: Seminário de Iniciação Científica, 15. São Luís-MA, p.23.

Boeck P, Falcão CAB, Leal PC, Yunes RA, Cechinel Filho V, Torres-Santos EC, Rossi-Bergmannc B 2006. Synthesis of chalcone analogues with increased antileishmanial activity. Bioorg Med Chem 14: 1538-1545.

Caldas AJM, Costa JML, Silva AAM, Vinhas V, Barral A 2002. Risk factors associated with asymptomatic infection by Leishmania chagasi in Northeast Brazil. T Roy Soc Trop Med H 96: 21-28.

Camacho MR, Phillipson JD, Croft LS, Kirby GC, Warhurst DC, Solis PN 2000. Terpenoids from Guarea rhophalocarpa. Phytochemistry 56: 203-210.

Camacho MR, Phillipson SL, Croft PN, Marshall SJ, Ghazanfar SA 2003. Screening of plants extracts for antiprotozoal and cytotoxic activities. $J$ Ethnopharmacol 89: 185191.

Carvalho PB, Ferreira EI 2001. Leishmaniasis phytotherapy. Nature's leadership against an ancient disease.
Fitoterapia 72: 599-618.

Chen M, Brogger Christensen S, Blom J, Lemmich E, Nadelmann L, Fich K, Theander TG, Kharazmi A 1993. Licochalcone A, a novel antiparasitic agent with potent activity against human pathogenic protozoan species of Leishmania. Antimicrob Agents Ch 37: 2550-2556.

Costa JML, Balby IAT, Rocha EJS, Silva ARS, Rebêlo JMM, Ferreira LA, Gama EA, Branco MRFC, Burattini MN, Soares NJS 1998. Estudo comparativo da leishmaniose tegumentar americana em crianças procedentes das áreas endêmicas de Buriticupu (Maranhão) e Corte de Pedra (Bahia)-Brasil. Rev Soc Bras Med Trop 31: 279-288.

Croft S, Coombs G 2003. Leishmaniasis, current chemotherapy and recent advances in the search for novel drugs. Trends Parasitol 19: 502-508.

Dhawan K, Dhawan S, Sharma A 2004. Passiflora: a review update. J Ethnopharmacol 94: 1-23.

Dujardin J-C 2006. Risk factors in the spread of leishmaniases: towards integrated monitoring? Trends Parasitol 22: 4-6

Fournet A, Barrios AA, Munõz V, Hocquemiller CA 1992. Effects of natural naphtoquinones in $\mathrm{BALB} / \mathrm{c}$ mice infected wifh Leishmania amazonensis and $L$. venezuelensis. Ann Trop Med Parasit 43: 219-222.

Fournet A, Ferreira ME, Rojas AA, Torres OS, Fuentes S, Nakayama H, Schinini A 1996. In vitro efficacy of oral and intralesional administration of 2-substituted quinolines in experimental treatment of new world cutaneous leishmaniasis caused by Leishmania amazonensis. Antimicrob Agents Ch 40: 2447-2451.

França F, Cuba CA, Moreira EA, Almeida M, Virgens ML, Marsden PD 1993. Avaliação do efeito do extrato de casca de cajueiro-branco (Anacardium occidentales) sobre a infecção por Leishmania (Viannia) braziliensis. Rev Soc Bras Med Trop 26:151-155.

França F, Lago EL, Marsden PD 1996. Plants used in the treatment of leishmanial ulcers due to Leishmania (Viannia) braziliensis in the endemic area of Bahia, Brazil. Rev Soc Bras Med Trop 29: 229-232.

Iwu MM, Jackson JE, Schuster BG 1994. Medicinal plants in the fight against leishmaniasis. Parasitol Today 10: 65-68.

Kam TS, Sim KM, Koyana T, Toyoshima M, Hayash M, Komiyama K 1997. Cytotoxic and leishmanicidal aminoglycosteroids and aminosteroids from Holarrhena curtisii. J Nat Prod 61: 1332-1336.

Kam TS, Sim KM, Koyano T, Toyoshima M, Komiyama K 1999. Leishmanicidal alkaloids from Kopsia griffithii. Phytochemistry 50: 75-79.

Kayser O, Kiderlen AF, Laatsch H, Croft SL 2000. In vitro leishmanicidal activity of monomeric and dimeric naphthoquinones. Acta Tropica 77: 307-314.

Loguercio AP, Battistin A, Castagna de Vargas A, Niura AH, Witt M 2005. Atividade antibacteriana de extrato hidro-alcoólico de folhas de jambolão (Syzygium cumini (L.) Skells) Ciência Rural 35: 371-376.

Mahiou V, Roblot F, Hocquemiller R, Cave A, Rojas de Arias A, Inchausti A, Yaluff G, Fournet A 1994. Aporphine alkaloids from Guatteria foliosa. J Nat Prod 57: 890895.

Martínez MA, Del P, Swain T 1993. Flavonoids and 
chemotaxonomy of the Commelinaceae. Biochem System Ecol 13: 391-402.

Martins LM 2002. Ocorrência da leishmaniose tegumentar e sua relação com impactos ambientais em Buriticupu, Amazônia do Maranhão. Dissertação (Saúde e Ambiente), Universidade Federal do Maranhão. Programa de Pós-Graduação em Saúde e Ambiente. São Luís. 70p.

Matos FJA 2000. Plantas medicinais: guia de seleção e emprego de plantas usadas em fitoterapia no Nordeste do Brasil. $2^{a}$ ed. Fortaleza:IU

Mathias LA, Emily A 1993. Tapping and Amazonian plethora: four medicinal plants of Marajó Island, Pará - Brazil. J Ethnopharmacol 40:53-75.

Michelin DC, Moreschi PE, Lima AC, Nascimento GGF, Paganelli MO, Chaud MV 2005. Avaliação da atividade antimicrobiana de extratos vegetais. Rev Bras Farmacogn 15: 316-320.

Ministério da Saúde (FUNASA) 2000. Manual de Controle da Leishmaniose Tegumentar Americana. Brasília: Centro Nacional de Epidemiologia, FUNASA, MS.

Mittra B, Saha A, Chowdhury AR, Pal C, Mandal S, Mukhopadhyay S, Bandypadhyay S, Majumder HK 2000. Luteolin, an abundant dietary component is a potent anti-leishmanial agent that acts by inducing topoisomerase ii-mediated kinetoplast DNA cleavage leading to apoptosis. Mol Med 6: 527-541.

Mittal N, Gupta N, Saksena S, Goyal N, Roy U, Rastogi AK 1998. Protective effect of picrolive from Pichrorhiza kurroa against Leishmania donovani infections in Mesocricetus auratus. Life Sci 63: 1823-1834.

Morais SM, Dantas JDP, Silva ARA, Magalhães EF 2005. Plantas medicinais usadas pelos índios Tapebas do Ceará. Rev Bras Farmacogn 15: 169-177.

Moreira RCR, Costa JML, Saldanha AC, Silva AR 1998. Projeto Buriticupu Maranhão II. Plantas usadas como terapêutica da leishmaniose tegumentar americana na região de Buriticupu-Maranhão. Rev Soc Bras Med Trop 31(Supl. 1): T248, 126.

Moreira RCR, Rebêlo JMM, Gama MEA, Costa JML 2002. Nível de conhecimento sobre Leishmaniose Tegumentar Americana (LTA) e uso de terapias alternativas por populações de uma área endêmica da Amazônia do Maranhão, Brasil. Cad Saúde Pub 18: 187-195.

Nakamura CV, Santos AO, Vendrametto MC, Luize OS, Dias Filho BP, Cortez DAG, Ueda-Nakamura T 2006. Atividade antileishmania do extrato hidroalcoólico e de frações obtidas de folhas de Piper regnellii (Miq.) C. DC. var. pallescens (C. DC.) Yunck. Rev Bras Farmacogn 16: 61-66.

Paula CDR, Sampaio JHD, Cardoso DRC, Sampaio RNR 2003. Estudo comparativo da eficácia de isotionato de pentamidina administrada em três doses durante uma semana e de $N$-metil-glucamina $20 \mathrm{mgSbV} / \mathrm{Kg} / \mathrm{dia}$ durante 20 dias para o tratamento da forma cutânea da leishmaniose tegumentar americana. Rev Soc Bras Med Trop 36:365-371.

Pereira RC, Oliveira MTR, Lemos GCS 2004. Plantas utilizadas como medicinais no município de Campos de Goytacazes - RJ. Rev Bras Farmacogn 14 (Supl. 1): $37-40$.

Pio Corrêa P 1984. Dicionário das plantas úteis do Brasil e das exóticas cultivadas. Imprensa Nacional, Rio de
Janeiro, vol. 5. Imprensa Nacional, Ministério da Agricultura, Rio de Janeiro, Brasil.

Queiroz EF, Roblot F, Cave A 1996. Pessoine and spinosine, two catecholic berberines from Annona spinescens. $J$ Nat Prod 59: 438-440.

Rath S, Trivelin LA, Imbrunito TR, Tomazela DM, Jesus MN, Marzal PC, Andrade Júnior HF, Tempone AG 2003. Antimoniais empregados no tratamento da leishmaniose: estado da arte. Quim Nova 26: 550553.

Rebêlo JMM, Oliveira ST, Barros VLL, Silva FS, Costa JML, Ferreira LAA, Silva AR 2000a. Plebotominae (Diptera: Psychodidae) de Lagoas, município de Buriticupu, Amazônia Maranhense. I - Riqueza e abundância relativa das espécies em área de colonização recente. Rev Soc Bras Med Trop 33: 1119.

Rebêlo JMM, Oliveira ST, Barros VLL, Silva FS 2000 b. Flebotomíneos da Amazônia Maranhense. IV Riqueza e abundância relativa das espécies em área de colonização antiga. Entomol Vect 7: 61-72.

Rebêlo JMM, Oliveira ST, Barros VLL, Silva FS, Costa JML, Ferreira LAA, Silva AR 2001a. Flutuações dos flebotomíneos (Diptera, Psychodidae) em área de colonização recente do Município de Buriticupu, Amazonia maranhense, Brasil. Rev Bras Entomol 45:11-16.

Rebêlo JMM, Oliveira ST, Silva FS, Costa JML 2001b. Sandflies (Diptera, Psychodidae) of the Amazonia of Maranhão. V. Seasonal occurrence ancient colonization area and endemic for cutaneous leishmaniasis. Rev Bras Biol 61:107-115.

Rego TJAS 1995. Fitogeografia de plantas medicinais do Maranhão. São Luis:Edufma. 133p.

Rocha LG, Almeida JRGS, Macêdo RO, Barbosa Filho JM 2005. A review of natural products with antileishmanial activity. Phytomedicine 12: 514-535.

Royo VA, Santos FF, Souza VA, Pereira AC, Da Silva R, Vinhólis AHC, Donate PM, Silva MLA, Albuquerque S, Bastos JK 2003. Biological activity evaluation of dibenzilbutirolactones lignans derivatives against Leishmania braziliensis. Rev Bras Farmacogn 13(Supl.): 18-21.

Sauvain M, Kunesch N, Poisson J, Gantier J-C, Gayral P, Dedet J-P 1996. Isolation of leishmanicidal triterpenes and lignans from Amazoniam liana Doliocarpus dentatus (Dellineaceae) Phytother Res 10:1-4.

Schmeda-Hirschmann G, Razmilic I, Sauvain M, Moretti C, Munoz V 1996. Antiprotozoal activity of jatrogrossidione from Jatropha grossidentada and jatrophone from Jatropha isabelli. Phytother Res 10: 375-378.

Sharma P, Rastori S, Bahatnager S, Srivastava JK, Dube A, Guru PY, Kulshreshtha DH, Dhawan BN 2003. Antileishmanial action of a plant Tephrosia purpurea against experimental visceral leishmaniasis. Drug Develop Res 60: 285-293.

Silva AR, Martins G, Melo GEN, Araújo P, Mendes MG 1979. Surto epidêmico de leishmaniose tegumentar americana ocorrido na colonização agrícola de Buriticupu (Estado do Maranhão), Brasil. Rev Inst Med Trop 21: 1-62

Silva SAG, Da Costa SS, Mendonça SCF, Silva EM, Moraes 
VLG, Rossi-Bergman B 1995. Therapeutic effect of oral Kalanchoe pinnata leaf extract in murine leishmaniais. Acta Tropica 60: 201-210.

Sindermann H, Croft SL, Engel KR, Bommer W, Eibl HJ, Unger C, Engel J 2004. Miltefosine (Impavido): the first oral treatment against leishmaniasis. Med Microbiol Immunol 193: 173-180.

Sousa MP, Matos MEO, Matos FJA, Machado MIL, Craveiro AA 1991. Constiuintes químicos de plantas medicinais brasileiras. Fortaleza:EUFC.

Suarez AI, Blanco Z, Delle Monache F, Compagnone RS, Arvelo F 2004. Three new glutarimide alkaloids from Croton cuneatus. Nat Prod Res 18: 421-426.

Tôrres AR, Oliveira RAG, Diniz MFFM, Araújo EC 2005. Estudo sobre o uso de plantas medicinais em crianças hospitalizadas da cidade de João Pessoa: riscos e benefícios. Rev Bras Farmacogn 15: 373-380.

Torres-Santos EC, Moreira DL, Kaplan MAC, Meirelles MN, Bergmann BR 1999. Selective effect of 2,6-dihydroxy4-methoxychalcone isolated from Piper aduncum on Leishmania amazonensis. Antimicrob Agents Ch 43: 1234-1241.

World Health Organization 2002. The leishmaniases and Leishmania/HIV co-infections. http://www.who. int/mediacentre/factsheets/fs $116 / \mathrm{en} /$. Consultado em 10/11/05. 